\title{
MULTIPLE-ACCESS TECHNOLOGY OF CHOICE IN 3GPP LTE \\ ${ }^{1}$ Ibikunle Frank, ${ }^{2}$ Dike Ike, ${ }^{3}$ Ajayi Jimi, ${ }^{4}$ Onasoga Kayode \\ $1,2,3,4$ Department of Electrical and Information Engineering Covenant University, Ota, Ogun state, Nigeria.
}

\begin{abstract}
Third-Generation Partnership Project (3GPP) standardizes an Evolved UMTS Terrestrial Radio Access Network (EUTRAN) as air interface in its release 8 LTE. Orthogonal Frequency Division Multiple Access(OFDMA) and Single CarrierFrequency Division Multiple Access(SC-FDMA)are key technologies for the air interface of mobile broadband systems.It is evident that mobile broadband access technologies are reaching a commonality in the air interface and networking architecture; they are being converged to an IP-based network architecture with OFDMA based air interface technology. The air interface of E-UTRAN is based on OFDMA in downlink and SC-FDMA in the uplink, making it possible to efficiently utilize bandwidth due to the orthogonally between sub-carriers and by assigning subsets of sub-carriers to individual users which allows for simultaneous data rate transmission from several users and differentiated quality of service for each user. In this paper, wehighlight the technologies behindOFDMA and SC-FDMA and also carry out performance comparison of the two air interface technologies. We brieflydescribe the 3GPP LTE standard, and its implementation using OFDMA and SC-FDMA technology.
\end{abstract}

Keywords- E-UTRAN, IP-based network, mobile broadband systems, OFDMA, SC-FDMA

\section{Council for Innovative Research}

Peer Review Research Publishing System

Journal: INTERNATIONAL JOURNAL OF COMPUTERS \& TECHNOLOGY

Vol 6, No 2

editor@cirworld.com

www.cirworld.com, member.cirworld.com 


\subsection{INTRODUCTION}

OFDMA was first introduced as an air interface technology for broadband wireless systems with the Wireless MANOFDMA air interface of IEEE 802.16d in 2004 [1]. A major development in communications networkswas born when the Third-Generation Partnership Project (3GPP) started its work to define a technical standard forthe Beyond 3G (B3G) systems. Release 8 of the 3GPP standard, which was finalized at the end of 2008, made a large technological gap with respect to previous releases and adopted OFDMA for the downlink and SC-FDMA for the uplink. The choice of SC-FDMA for the uplink was motivated by the limited Peak-to-Average-Power Ratio of this technique.The undesirable high PAPR of OFDMA in the downlink led 3GPP to choose a different modulation format for the LTE uplink. Presently, there is a debate in the wireless community on which of the two technologies is the best.

In 2010, Ciochina and Hikmet [2]published a paper, which reviewed both OFDMA and SC-FDMA technologies and did a performance evaluation of these two multiple access technologies, and concluded that OFDMA can be expected to offer a higher cell capacity, while SC-FDMA can lead to cell range extension.LTE, whose radio access is called Evolved UMTS Terrestrial Radio Access Network (E-UTRAN), has substantially improved end-user throughputs, capacity and it has reduced user latency, thus users are now able to have amuch improved mobile communication. LTE uses Internet Protocol for carrying all types of traffic, and thus provides improved Quality of Service.

This paper focuses on one of the main applications of OFDMA and SC-FDMA technology as air interface technologies for LTE mobile broadband systems. The outline of this paper is as follows:Section II gives the description of the principles of OFDMA and SC-FDMA as the multiple access system of choice for $4 \mathrm{G}$ systems. Section III gives a highlight of LTE system features and architecture. Section IV gives full details of the use of OFDMA and SC-FDMA technologies in the implementation of 3GPP LTE and also compares the two technologies.

While in Section $\mathrm{V}$, we draw the conclusion for the paper.

\subsection{SYSTEM IMPLEMENTATION OVERVIEW}

LTE air-interface is implemented using OFDMA and SC-FDMA. In this section, the use of the above stated technologies in the implementation of LTE system is described.

\subsection{Principles of OFDMA}

Orthogonal Frequency-Division Multiple Access (OFDMA) is a multi-user version of theOrthogonal Frequency-Division Multiplexing(OFDM) digital modulation scheme. OFDM is a form of modulation technique that uses a large number of closely spaced sub-carriers that are modulated with low rate data. The key principle in OFDM is the use of orthogonal subcarriers for sending several data symbols in parallel resulting in better spectral efficiencies and simpler equalization methods at the receiver. The samples of the transmitted OFDM signal are obtained by performing an Inverse Fast Fourier Transform (IFFT) operation on the group of data symbols to be sent on orthogonal sub-carriers [2]. The recovery of the data symbols from the orthogonal sub-carriers is accomplished using a Fast Fourier Transform (FFT) operation on a block of received samples. The wideband wireless radio channel with frequency-selective fading is thus turned into a set of narrow-band channels (subcarriers) with flat fading. Each data symbol is then transmitted on one subcarrier, making it resilient against multipath propagation [3]. These carrier signals would normally be expected to interfere with each other, but by making the sub-carriers orthogonal to each other there is no mutual interference. This is achieved by having the carrier spacing equal to the reciprocal of the symbol period. OFDMA is similar to OFDM in function, with the main difference being that instead of being allocated all of the available subcarriers, the base station allocates a subset of carriers to each user in order to accommodate multiple transmissions simultaneously. The subcarrier mapping is the key in implementing OFDMA because it assigns users to subcarriers.In general, there are two ways of assigning users to subcarriers: distributed subcarriers and contiguous subcarriers. A logical set of subcarriers is sometimes called a subchannel,and eachuser may be assigned one or more sub-channels. The block diagram of OFDMA is as shown in Figure 1.

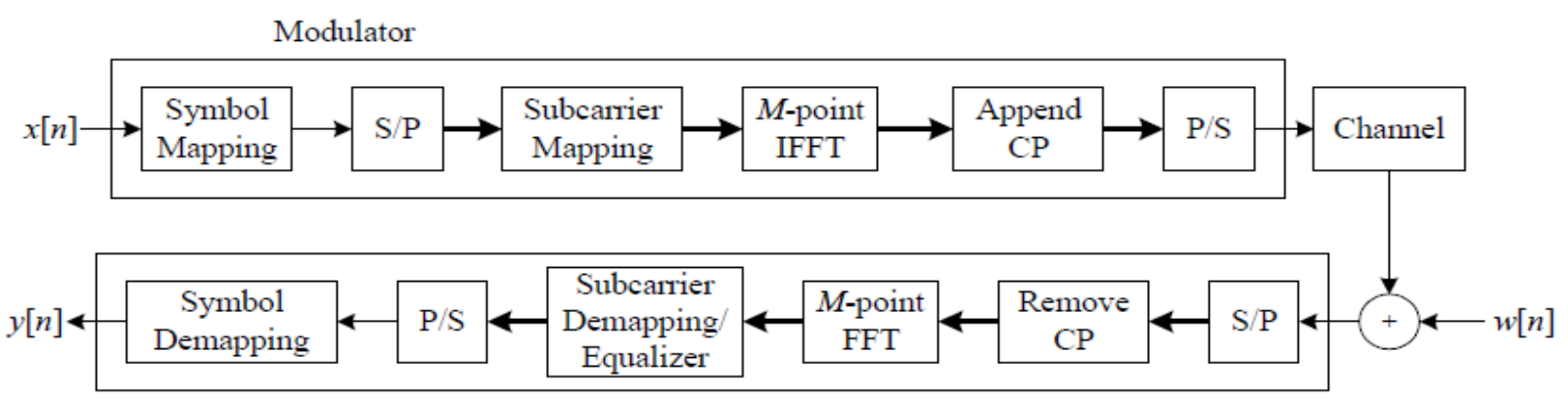

Demodulator

Figure 1: Block Diagram of OFDMA [10]

OFDMA may exploitmultiuser diversity by assigning the subcarriers according to channel quality of eachuser if channel side information is present in the transmitter. Also, modulation andpower control may be fine tuned according to each subcarrier with respect to channelcondition.OFDMA scheduling may also be based on frequency diversity. An 
orthogonalcode may be defined for each user to determine their hopping pattern across timeand frequency. This way, fading of a user may be averaged over without any channelinformation. Also, in a typical cell deployment, same frequency is used in multiplespatially located cells. OFDMA may reduce the interference coming from the adjacentcells operating with the same frequency by assigning different sets of orthogonalcodes in each cell. This may lead to utilize OFDMA to deploy a single frequency network in several ways. In one way, the same frequency is used by the base stations but slot allocation is done with respect to a code that is user specific. Codes need not to be strictly orthogonal, which would otherwise result in low capacity system, since users are separated in distance, which makes the probability of interference minimal.

The baseband structure of a generalized multi-carrier transmitter, which applies to all types of single carrier or multi carrier modulation signals transmitted in blocks is as shown in the Figure 2.

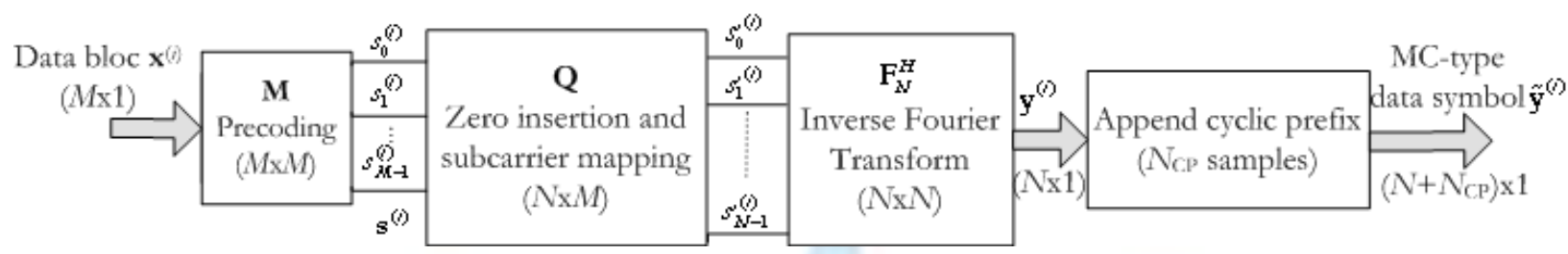

Figure 2:Baseband Structure of a Generalized Multi- Carrier Transmitter [4]

Let us denote by $\mathrm{xk}(\mathrm{i})$ the information symbols which are parsed into data blocks $\mathrm{x}$ (i) of sizeM. Data blocks belonging to a certain user are pre-coded withan $M \cdot M$ matrix $M$. The user-specific $M$-sized output $s(i)$ is then mapped onto a set of $M$ out of $\mathrm{N}$ inputs of the inverse discrete Fourier transform (IDFT) conveniently chosen by the user-specific subcarrier mapping $\mathrm{N}$ - M matrix Q. FN and FHN stand for the N-point direct and inverse normalized DFT matrices, respectively. A cyclic prefix which needs to be longer than the largest multipath delay is usually inserted before transmission to eliminate the intersymbol interference arising from multipath propagation. In this general representation, OFDMA corresponds to the case without pre-coding, i.e., the pre-coding matrix corresponding to OFDMA is the identity matrix $(M=I M)$. The IDFT operation is equivalent to splitting the information into $M$ parallel data streams that are transmitted by modulating $M$ out of the $N$ distinct subcarriers equally spaced in the channel bandwidth. Thus, OFDMAconsists of assigning different subcarrier groups of an OFDM symbol to different users[4].

\subsection{Principles of SC-FDMA}

The block diagram representation of SC-FDMA is as shown in Figure 3. The data symbols are first modulated by a base band modulator resulting in a sequence of modulated complex symbols. The next serial modulated data is then converted into $\mathrm{N}$ parallel data streams. An Npoint Discrete Fourier Transform (DFT) is used to transformthe time domain modulated symbols to frequency domainsymbols. After that, a sub-carrier mapping is used to map N DFToutput symbols to one of $\mathrm{M}$ orthogonal sub-carriers, where $\mathrm{M}>\mathrm{N}$ isthe number of orthogonal frequency sub-carriers, and $\mathrm{N}$ should be an integer multiple of Mso that $M=N^{\star} Z$ where $Z$ is maximum number of users that can be supported by asystem. After this, an Inverse DFT (IDFT) is performed which transforms thecomplex frequency domain symbols to time domain signals. Each symbol is modulated by a single high frequencycarrier and transmitted sequentially.After that the Cyclic Prefix (CP) is added by prefixing symbols with a copy of the end of the symbol as aguard interval to eliminate the Inter Symbol Interference .To enable equalization, the length of Cyclic Prefix should be minimum equal to maximum delay in the channel. Before modulating the signal with high frequency, a pulse shaping filter is used to shape the signal to get the desired spectrum.

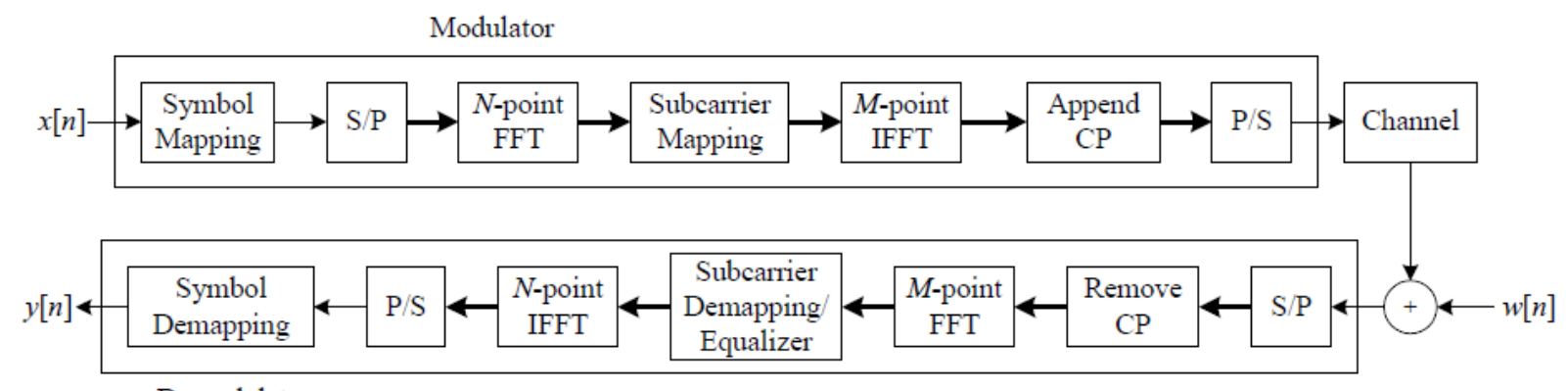

Demodulator

Figure 3: Block Diagram of the Transmitter and Receiver of SC-FDMA[10]

At the receiver side we do the inverse of what we have done at transmitter. First we demodulate our signal to a lower frequency then we remove Cyclic Prefix. After removing the $\mathrm{CP}$, the receiver transforms the received signal into frequency domain with the help of DFT. It then de-maps the sub-carriers and then performs frequency domain equalization. The equalized signal is then transformed to time domain by IDFT and signal detection is done in time domain .SC-FDMA is an extension of SC-FDE that allows multiple access with a complexity similar to that of OFDMA. Both technologies use almost the same transceiver blocks, being the DFT pre-coding and inverse pre-coding stages, which are added in SCFDMA at transmitter and receiver ends, the main difference between them. Due to those blocks, SC-FDMA has better 
capabilities in terms of envelope variations of the transmitted signal and, therefore, its PAPR is lower (up to $2 \mathrm{~dB}$ ) than in OFDMA [6]. That leads to greater efficiency in power consumption, a desirable feature in user equipment. In addition, SCFDMA complexity focuses on the receiverend, hence, it is an appropriate technology for uplink transmission since complexity at the base station is not an issue. Due to these features, SC-FDMA was chosen as the medium access technique for 3GPP LTE uplink. Note that the DFT operation in SC-FDMA spreads the energy of one sub-carrier over all allocated sub-carriers before the IFFT.

\subsection{LTE SYSTEM AND ARCHITECTURE}

LTE is a standard developed by 3GPP for mobile data communications and an evolution of the GSM/UMTS standards. The goal of LTE was to increase the capacity and speed of wireless data networks using advanced digital signal processing and modulationtechniques. Another goal was to transform the network architecture to an IP-based system, which significantly reduced latency compared to the $3 G$ architecture. The LTE specification provides downlink peak rates of $300 \mathrm{Mbit} / \mathrm{s}$, uplink peak rates of $75 \mathrm{Mbit} / \mathrm{s}$ and Quality of Service provisions permitting a transmissionlatency of less than $5 \mathrm{~ms}$. LTE supports multi-cast and broadcast streams. LTE carrier bandwidths are scalable and range from $1.4 \mathrm{MHz}$ to $20 \mathrm{MHz}$. In LTE, there is provision for both Frequency Division Duplexing(FDD)and Time Division Duplexing(TDD). The IP-based network architecture, called the Evolved Packet Core (EPC) was designed to replace the GPRS Core Network, it supports handovers for both voice and data to cell towers with older network technology such as GSM, UMTS and CDMA2000. The simpler architecture results in lower operating costs, for example, each EUTRAN cell can support up to four times the data and voice capacity supported by HSPA.

Evolved-UTRAN is the air interface of LTE. Its main features are:Peak down-load rates up to $300 \mathrm{Mbit} / \mathrm{s}$ and upload rates up to $75 \mathrm{Mbit} / \mathrm{s}$ depending on the user equipment category; Low data transfer latencies (sub-5 ms latency for small IP packets in optimal conditions), lower latencies for handover and connection setup time than with previous radio access technologies; Improved support for mobility, exemplified by support for terminals moving at up to $300 \mathrm{~km} / \mathrm{h}$ or $450 \mathrm{~km} / \mathrm{h}$ depending on the frequency band;OFDMA is used for the downlink, while SC-FDMA is used for the uplink for efficient power conservation;There isincreased spectrum flexibility: $1.4 \mathrm{MHz}, 3 \mathrm{MHz}, 5 \mathrm{MHz}, 10 \mathrm{MHz}, 15 \mathrm{MHz}$ and $20 \mathrm{MHz}$ wide cells are standardized. LTE supports cell sizes from tens of metres radius to $100 \mathrm{~km}$ radius macrocells. In city and urban areas, higher frequency bands are used to support high speed mobile broadband, in this case, cell sizes may be $1 \mathrm{~km}$ or even less; LTE architecture is very simplified, the network side of E-UTRAN is composed only of eNodeBs and it enables service providers to reduce the cost of owning and operating the network by allowingthe service providers to have separate core network (MME, SGW, PDN GW) while the Evolved UMTS Terrestrial Radio Access NetworkeNBs is jointlyshared by them. This is enabled by the S1-flex mechanism that enables each eNB to be connected to multiplecore network entities. When a User Equipment (UE) attaches to the network, it is connected to the appropriate core network entities based on theidentity of the service provider sent by the User Equipment[7].

The system architecture of the LTE consists of the following functional elements as shown in Figure 4.

Evolved Radio Access Network (RAN): The evolved RAN for LTE consists of a single node the eNB that interfaces with the User Equipment. The eNB hosts the Physical, Medium Access Control, Radio Link Control, and Packet Data Control Protocol layers which include the functionality of user-plane header-compression and encryption. It also provides Radio Resource Control (RRC) functionality that corresponds to the control plane.

Serving Gateway (SGW):The Serving gateway provide routing and forwarding of users's data packets, and also, acts as the mobility anchor for the user plane during inter-eNB handovers, and as the mobility anchor between LTE and other 3GPP technologies. For idle state User Equipment, the Serving Gate Way stops the DL data path and enables paging.

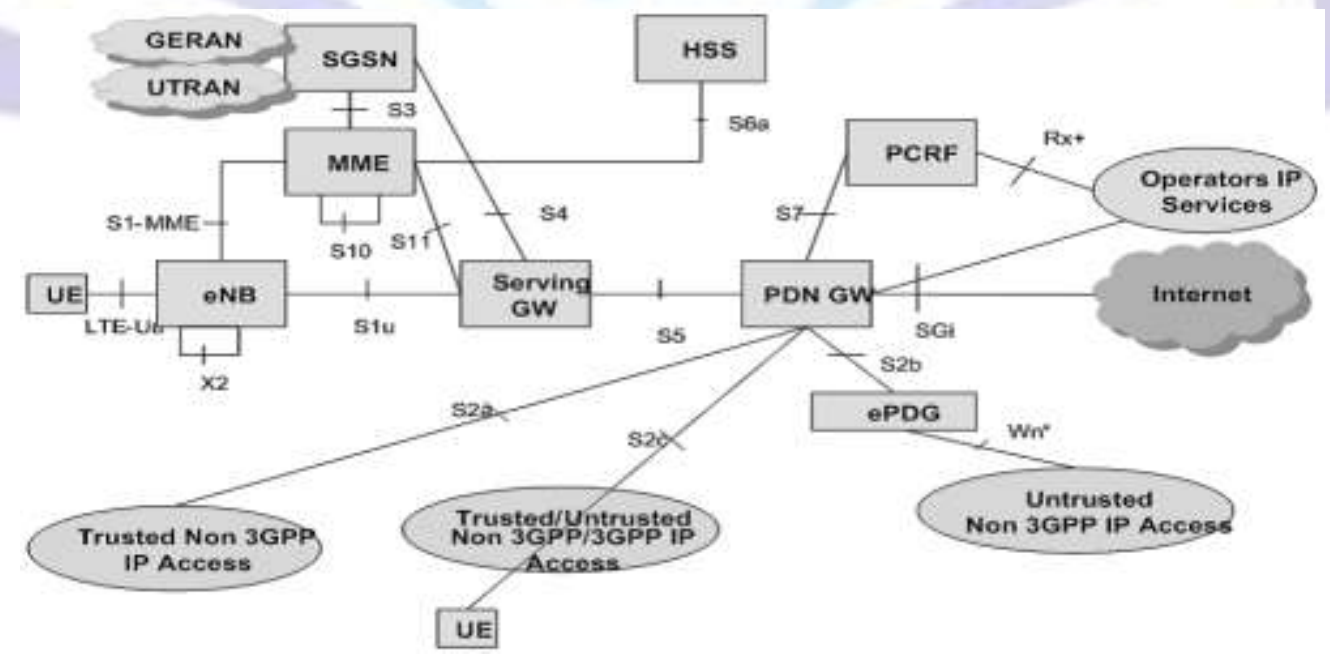

Figure 4:LTE system architecture [7] 
Mobility Management Entity (MME):The mobility Management Entity is a control node for the LTE access network. It is involved in the bearer activation or deactivation process and chooses the SGW for a UE at the initial attach and at time of intra-LTE handover involving Core Network $(C N)$ node relocation. It is responsible for idle mode UE tracking and paging procedure including retransmissions.It is involved in the bearer activation/deactivationprocess and is also responsible for choosingthe SGW for a UE at the initial attach and at timeof intra-LTE handover involving Core Network (CN)node relocation. It is responsible for authenticatingthe user (by interacting with the HSS).

Packet Data Network Gateway (PDN GW):The connectivity between the User Equipment (UE) and external packet data networks is being provided by PDN GW. It is the point of exit and entry of traffic for the UE. A UE may have simultaneous connectivity with multiple PDN GW In order to access multiple Packet Data Networks. The PDNGW also performs policy enforcement, packet filteringfor each user, and packet screening. The PDNGW acts as the anchor for mobility between3GPP and other technologies

\subsection{UPLINK AND DOWNLINK ACCESS SCHEMES IN LTE}

One of the key elements of LTE is the use of OFDMA (Orthogonal Frequency Division Multiple access) and SC-FDMA (Single carrier Frequency Division Multiple Access) as its air interface technology. OFDMA is used in a number of other systems from WiMAX to broadcast technologies, including Digital Video Broadcasting and Digital Audio Broadcasting. OFDMA has many advantages including its robustness to multipath fading and interference. In addition to this, even though, it may appear to be a complicated form of modulation, it lends itself to digital signal processing techniques. SCFDMA combines the advantages of OFDMA with lower peak-to-average-power ratio.In view of their advantages, OFDMA and SC-FDMA are natural choices for the new LTE cellular standard.

\subsection{Downlink Access Scheme in LTE}

OFDMA as implemented in LTE allocates a number of time/frequency resources to specific users. The time/frequency resource grid is subdivided into logical basic resource units which consist of sub-bands in frequency and one or more OFDM symbols in time domain. A sub-band comprises several subcarriers. The basic resource units are then mapped to the physical OFDMA frame. If the subcarriers and symbols are permuted in frequency and optionally additionally in time (distributed scheme), the data is spread over the carrier frequency, such that frequency diversity is exploited. This reduces the probability that a whole data block is dropped due to frequency selective fading or interference in a sub-band. In contrast, in the contiguous permutation scheme the logical resource units are mapped one to one to the corresponding physical subcarriers. This allows for exploitation of multi-user diversity by frequency-selective scheduling. However, intercell interference must then be avoided by frequency partitioning or by coordination schemes. LTE specifies these permutation schemes. However, currently only the distributed permutation scheme is deployed in commercial networks due to its lower requirements on channel feedback and lower scheduling complexity.

The OFDM signal used in LTE comprises a maximum of 2048 different sub-carriers having a spacing of $15 \mathrm{kHz}$. Although it is mandatory for the mobiles to have capability to be able to receive all 2048 sub-carriers, not all need to be transmitted by the base station which only needs to be able to support the transmission of 72 sub-carriers. In this way all mobiles will be able to talk to any base station.Within the OFDM signal it is possible to choose between three types of modulation, namely; QPSK, 16 QAM, and 64 QAM. The exact format is chosen depending upon the prevailing conditions. The lower forms of modulation, (QPSK) do not require such a large signal to noise ratio but are not able to send the data as fast. Only when there is a sufficient signal to noise ratio can the higher order modulation format be used.In the downlink, the subcarriers are split into resource blocks. This enables the system to be able to compact the data across standard numbers of subcarriers.Resource blocks comprise 12 subcarriers, regardless of the overall LTE signal bandwidth. They also cover one slot in the time frame. This means that different LTE signal bandwidths will have different numbers of resource blocks[8].

In OFDMA networks, cell edge users are constrained byinterference. In contrast to CDMA systems, macrodiversityschemes like soft-combining are not used in LTE.Instead, interference mitigation schemes areused. The most basic schemes are simple frequency reuseschemes together with frequency diversitypermutations. In frequency reuse, the available spectrum isinto split into fractions which are then assigned to cellssuch that the distances between cells with the same spectrumpartition are maximized. In conventional frequency reuse schemes, thefrequency reuse factor corresponds to the number of partitions.

However, conventional frequency reuse schemes divide theavailable spectrum in fixed parts which are independent of theactual received interference at the mobile stations. This leads to lower peak capacities for users at the cell center. Fractional frequency reuse or soft frequency reuse schemes areaddressing this problem by assigning the whole frequencyspectrum to user at the cell center, while maintaining a higher-orderfrequency reuse scheme for cell-edge users. In LTE, this isachieved by different power allocations across the frequency dimension. One of themain challenges here is to decide which mobile devices areassigned to which frequency bands.

OFDMA downlink implements downlink physical channels in order to convey information from higher layers. Scheduler in eNB allocates resource blocks to users for a predetermined amount of time. Downlink resource block, is the smallest element of resource allocation, where 12 subcarriers constitute a resource block with one downlink slot. LTE frames are $10 \mathrm{~ms}$ in duration and they are divided into ten sub-frames where each sub-frame is further divided into two slots, each of 0.5 -ms duration. Slots consist of either 6 or 7 OFDM symbols depending on whether the normal or extended cyclic prefix is employed. Longer cyclic prefix is desired to address longer fading that can be encountered in multi-cell broadcast service or very large cell deployments. 
Parameters for OFDMA downlink are shown in Table 1 for system bandwidths ranging from 1.25 to $20 \mathrm{MHz}$. The number of available subcarriers changes depending on the transmission bandwidth; however, OFDM downlink transmission scheme uses fixed subcarrier spacing regardless of transmission bandwidth. Basic downlink data-modulation schemes are QPSK, 16QAM, and 64QAM. Alternative enhanced modulation scheme is OFDM with pulse shaping (OFDM/OQAM), which does not require a guard interval. Pilot symbols, which are the physical signals that terminate or originate in the physical layer, are used to estimate the channel impulse response and for time and frequency synchronization. A pseudorandom numerical sequence is used in order not to encounter interference. A specific set out of 510 different orthogonal sequences is assigned to each cell in order to differentiate a cell from others

Table1:Parameters for downlink transmission scheme for OFDMA in LTE

\begin{tabular}{|l|l|l|l|l|l|l|}
\hline Transmission Bandwidth(MHz) & 1.25 & 2.5 & 5 & 10 & 15 & 20 \\
\hline Sub-frame duration (ms) & 0.5 & 0.5 & 0.5 & 0.5 & 0.5 & 0.5 \\
\hline Sub-carrierspacing (KHz) & 15 & 15 & 15 & 15 & 15 & 15 \\
\hline Number of available Resource Blocks & 6 & 12 & 25 & 50 & 75 & 100 \\
\hline Sampling Frequency (MHz) & 1.92 & 3.84 & 7.68 & 15.36 & 23.04 & 30.72 \\
\hline FFT Size & 128 & 256 & 512 & 1024 & 1536 & 2048 \\
\hline No. of occupied sub-carriers & 76 & 151 & 301 & 601 & 901 & 1201 \\
\hline Resource Blocks Bandwidth (KHz) & 180 & 180 & 180 & 180 & 180 & 180 \\
\hline
\end{tabular}

\subsection{Uplink Access Scheme in LTE}

SC-FDMA combines the properties of SC transmission with an OFDMA-like multiple access and attempts to take advantage of the strengths of both techniques: Low PAPR and flexible dynamic frequency allocation. For the LTE uplink, SC-FDMA is used for the access technique. In SC-FDMA, data symbols are pre-coded with a discrete Fourier transformation, such that each data symbol is transmitted in parallel over a group of subcarriers. The resulting timedomain waveform resembles single-carrier waveforms with lower PAPR.

One of the key parameters that affect all mobiles is that of battery life. Even though battery performance is improving all the time, it is still necessary to ensure that the mobiles use as little battery power as possible. With the RF power amplifier that transmits the radio frequency signal via the antenna to the base station being the highest power item within the mobile, it is necessary that it operates in as efficient mode as possible. This can be significantly affected by the form of radio frequency modulation and signal format. Signals that have a high peak to average ratio and require linear amplification do not lend themselves to the use of efficient RF power amplifiers. As a result it is necessary to employ a mode of transmission that has as near a constant power level when operating. Unfortunately OFDMA has a high peak to average ratio. While this is not a problem for the base station where power is not a particular problem, it is unacceptable for the mobile. As a result, LTE uses a modulation scheme known as SC-FDMA which is a hybrid format. This combines the low peak to average ratio offered by single-carrier systems with the multipath interference resilience and flexible subcarrier frequency allocation that OFDMAprovides.

In uplink, data are mapped onto a signal constellation that can be QPSK, 16QAM, or 64QAM with respect to channel quality. Modulated QPSK/QAM symbols are not directly used but sequentially fed into an FFT block to obtain the discrete frequency representation of the QPSK/QAM symbols. The FFT block is then mapped to an IFFT block to convert the sequence into time domain. This is to reduce the PAPR in the time domain since uncontrollable fluctuations are now restricted to the frequency domain because individual subcarrier amplitudes can actually vary more in the frequency domain as compared to OFDM. In brief, first FFT introduces high PAPR but second IFFT smoothes that over. Parameters for SC-FDMA uplink are shown in Table 2 for system bandwidths ranging from 1.25 to $20 \mathrm{MHz}$.

Table 2: Parameters for uplink transmission scheme for SC-FDMA in LTE

\begin{tabular}{|l|l|l|l|l|l|l|}
\hline $\begin{array}{l}\text { Transmission } \\
\text { Bandwidth } \\
\text { (MHz) }\end{array}$ & 1.25 & 2.5 & 5 & 10 & 15 & 20 \\
\hline $\begin{array}{l}\text { Sub-Frame } \\
\text { duration (ms) }\end{array}$ & 0.5 & 0.5 & 0.5 & 0.5 & 0.5 & 0.5 \\
\hline $\begin{array}{l}\text { Long } \\
\text { block/Short } \\
\text { blockFFT size }\end{array}$ & $128 / 64$ & $256 / 128$ & $512 / 256$ & $1024 / 512$ & $1536 / 768$ & $2048 / 1024$ \\
\hline $\begin{array}{l}\text { Long } \\
\text { block/Short }\end{array}$ & $66.67 / 33.33$ & $66.67 / 33.33$ & $66.67 / 33.33$ & $66.67 / 33.33$ & $66.67 / 33.33$ & $66.67 / 33.33$ \\
\hline
\end{tabular}




\begin{tabular}{|l|l|l|l|l|l|}
\hline block size(s) & & & & & \\
\hline $\begin{array}{l}\text { Number of } \\
\text { available } \\
\begin{array}{l}\text { Resource } \\
\text { blocks. }\end{array}\end{array}$ & & 6 & 12 & 24 & 36 \\
\hline
\end{tabular}

\subsection{Comparison Analysis of OFDMAand SC-FDMAin LTE}

OFDMA utilizes narrow-band orthogonal subcarriers and creates multiple data streams. The transmission rate in each subcarrier is inversely proportional to the total number of orthogonal subcarriers. Number of subcarriers (M) depends on the available bandwidth and could be 512, 1,024, or more. As a result, OFDMA system transmits information on $M$ orthogonal subcarriers, each operating bit rate of $1 / \mathrm{M}$ fold bit rate of the originalsignal. This rate decrease helps to alleviate the multipath effect of the channel and reduces the equalizer complexity in the receiver. OFDMA has some weaknesses, among others: the high peak-to-average power ratio (PAPR) is still one of the key drawbacks in the transmitted OFDMA signals [9]. This is due to unpredictable envelope fluctuations after IFFT. SC-FDMA spreads the energy of one subcarrier over all subcarriers before the IFFT. This way spectral nulls in the channel is reduced with averaging. Hence, PAPR is reduced. This subtle idea is performed by introducing additional FFT block before the IFFT block of the transmitter as seen. In OFDMA, first, information bits are converted to complex numbers with modulation, then, the complex numbers are mapped to IFFT block of length $M$ whereeach number stream is transmitted in a subcarrier out of M. This could be seen as anindependent transmission block, and each block produces a time domain signal thatare transmitted simultaneously. IFFT block performs these steps and converts thesedifferent signal streams from frequency domain into a time domain signal. In uplinkOFDMA, of course, each mobile station only uses $n$ subcarriers out of $M$ and leavesthe rest null in IFFT process.

In SC-FDMA, these complex numbers are first sent to additional N-point FFTblock in order to spread the energy over all the subcarriers. We know that FFT multiplieseach complex number with a multiplier and introduces $\mathrm{N}$ complex numbers. As a result, output of FFT block is considered as modified complex numbers, andeach output contains a portion of every input number. These new modified numbersare sent to M-point IFFT block as in OFDMA. Note that $N<M$ and as in OFDMA,zeros are sent in the unoccupied subcarriers.In the receiver side, OFDMA utilizes a simple equalizer per subcarrier afterFFT. But, SC-FDMA utilizes a complex equalizer before sending the resultant tolFFT. IFFT removes the effect of the FFT in the transmitter. Notice that result of theIFFT is again a time domain signal; the time domain signal is sent to a single detectorto create the bits.SC-FDMA receiver is more complex than OFDMA, but in the transmittersimpler power amplifiers can be utilized to reduce the power consumption. Thesefortify the SC-FDMA as an uplink transmission scheme, since power efficiency andcomplexity is important for mobile stations but not in the base station. Table 3 summarizes thedifferences between the multiple access technologies. When analyzed one subcarrier at a time, OFDMA resembles the original data symbols. At full bandwidth, however, the signal looks like Gaussian noise in terms of its PAPR statistics and the constellation. The opposite is true for SC-FDMA. Its relationship to the original data symbols is evident when analyzing the entire signal bandwidth whereupon the constellation (and hence low PAR) of the original data symbols can be observed rotating at $\mathrm{N}$ times the SC-FDMA symbol rate.

Table 3:Comparisonbetween OFDMA and SC-FDMA

\begin{tabular}{|c|c|c|}
\hline Metrics & OFDMA(Downlink) & SC-FDMA (Uplink\} \\
\hline Mapping & Map Input bits to Frequency Symbols & Map Input bits to Time Symbols \\
\hline $\begin{array}{l}\text { Peak-to-average Power } \\
\text { Ratio }\end{array}$ & High PAPR (Gaussian) & Low PAPR \\
\hline Sensitivity & Higher Sensitivity to Frequency Offset & $\begin{array}{l}\text { Lower Sensitivity to Frequency } \\
\text { Offset }\end{array}$ \\
\hline $\begin{array}{l}\text { Information Carried by } \\
\text { Sub-carrier }\end{array}$ & $\begin{array}{l}\text { Each Sub-carrier only carries information } \\
\text { related to one specific symbol }\end{array}$ & $\begin{array}{l}\text { Each } \\
\text { information of all transmitted } \\
\text { symbols }\end{array}$ \\
\hline $\begin{array}{l}\text { Observable } \\
\text { Constellation }\end{array}$ & Not equal to Data Symbol & Equal to Data Symbol \\
\hline Complexity & $\begin{array}{l}\text { Less Complex to Implement, does not } \\
\text { possess DFT pre-coding }\end{array}$ & $\begin{array}{l}\text { More Complex to Implement due to } \\
\text { additional DFT Pre-coding. }\end{array}$ \\
\hline Transmission Scheme & Double stream Per Antenna Rate Control & $\begin{array}{l}\text { Single stream Per Antenna Rate } \\
\text { Control }\end{array}$ \\
\hline
\end{tabular}

\subsection{CONCLUSION}


In this paper, we have been able to describe the principles of operation of OFDMA and SC-FDMA and their application in the implementation of LTE technology, and we have done a comparative analysis of the two technologies. Today, there is an SC-FDMA vs. OFDMA debate in wireless communications. Whereas OFDMA was selected by the WiMAX Forum for mobile WiMAX systems for both downlink and uplink, the 3GPP project preferred to use OFDMA for the downlink only and favored SC-FDMA for the uplink. We also described the system features and architecture of the 3GPP LTE, and implementation in LTE using the two access technologies. LTE is an optimized mobile OFDMA and SC-FDMA solution that continues 3G's track record of mobility and high spectral efficiency, and it is currently the mobile broadband of choice worldwide.

\section{REFERENCES}

[1] IEEE, "IEEE Std 802.16-2004, Air Interface for Fixed Broadband Wireless Access Systems", 2004.

[2]lbikunle, F.A., "Broadband Wireless Access Solution based on OFDMA Technique in WiMAX" IEEE 802.16,Pacific Journal of Science and Technology, 10 (1), 2009, 286-294.

[3] A. Maeder, N. Zein, "OFDMA in the Field: Current and Future Challenges",ACM SIG COMM Computer Communication Review, 40 (5), 2010, 71-76.

[4]ChristinaCiochina, Hikmet Sari, "A Review of OFDMA and Single- Carrier FDMA and some Recent

Results",Advances in Electronics and Telecommunications, 1(1), 2010, 35-40.

[6]"Evolution of LTE". LTE World. Retrieved October 24, 2011

[7]"Long Term Evolution (LTE): A Technical Overview". Motorola.Retrieved July 3, 2010.

[8]www.radio-electronics.com.

[9]Imran Baig, VarunJeoti. , PAPR Reduction in Layered OFDMA of LTE-A: A New Precoding Adaptive Uplink RA System,Journal of Engineering Science and Technology, 7(1), 2012, 131-141.

[10] NimaSoltani, "Comparison of Single-Carrier FDMA vs. OFDMA as 3GPP Long-Term Evolution Uplink", unpublished, 2009. 